\title{
On the Study of Autonomous Underwater Vehicles by Computational Fluid-Dynamics
}

\section{João Victor Nunes de Sousa ${ }^{1 *}$, Antonio Gilson Barbosa de Lima1, Francisco Alves Batista ${ }^{2}$, Edna Cordeiro de Souza ${ }^{3}$, Daniel César de Macedo Cavalcante ${ }^{4}$, Paulo de Morais Pessôa1, João Evangelista Franco do Carmo ${ }^{1}$}

\footnotetext{
${ }^{1}$ Department of Mechanical Engineering, Federal University of Campina Grande, Campina Grande, Brazil ${ }^{2}$ Department of Physics, State University of Paraíba, Campina Grande, Brazil

${ }^{3}$ Department of Physics and Mathematical, Federal University of Campina Grande, Campina Grande, Brazil ${ }^{4}$ Federal Institute of Education, Science and Technology of the Sertão Pernambucano, Serra Talhada, Brazil Email: *joao.vns@hotmail.com
}

How to cite this paper: de Sousa, J.V.N., de Lima, A.G.B., Batista, F.A., de Souza, E.C., de Macedo Cavalcante, D.C., de Morais Pessôa, P. and do Carmo, J.E.F. (2020) On the Study of Autonomous Underwater Vehicles by Computational Fluid-Dynamics. Open Journal of Fluid Dynamics, 10, 63-81. https://doi.org/10.4236/ojfd.2020.101005

Received: February 6, 2020

Accepted: March 13, 2020

Published: March 16, 2020

Copyright $\odot 2020$ by author(s) and Scientific Research Publishing Inc. This work is licensed under the Creative Commons Attribution International License (CC BY 4.0).

http://creativecommons.org/licenses/by/4.0/

\begin{abstract}
Autonomous Underwater Vehicles (AUV's) are considered as advanced classes of vehicles, capable of performing pre-established missions without physical communication with the ground or human assistance. The research and development of this type of vehicles have been motivated, due to its excellent characteristics, ideal to the military, scientific and industrial sectors. Thus, the objective of this paper is to study fluid flow behavior past over AUV's, without and with control surfaces (rudders), by Computational Fluid-Dynamics (CFD), aiming to obtain information about the impact of the operating depth and control surfaces on the vehicle's hydrodynamics, in order to help researchers and designers of this class of vehicles. Results of the drag coefficient, pressure, velocity and streamlines distribution around the vehicles are presented and analyzed.
\end{abstract}

\section{Keywords}

Autonomous Underwater Vehicle, Fluid Flow, Computational Fluid-Dynamics, Drag Coefficient

\section{Introduction}

An Autonomous Underwater Vehicle, or AUV, can be defined as a vehicle that travels submerged, without physical communication with the ground and without the need of a human operator. The AUV's are included in Unmanned Underwater Vehicles group, well better known as UUV's. 
During the last years research and development of AUV's have increased significantly because of the extremely favorable characteristics they have, such as the ability to operate autonomously in hostile environments [1] [2], such as unexplored or contaminated areas, enemy territorial seas or deep waters. All ways of using AUVs are very interesting for the military, scientific and industrial sectors.

The application of AUV's has been growing by the constants technological advances, mainly in electronics and robotics, allowing the execution of high precision missions, the reduction in the costs of design and operation, and the increase in embedded equipments quality, as well as for new technologies of batteries and power management, enabling the increase in autonomy, maintenance and safety in operations with this class of vehicles [3].

The use of numerical techniques to solve engineering problems is nowadays a reality due to the development of high processing capacity computers. These techniques have many advantages, being able to solve complex problems (which exact solutions can't be obtained and often can't be reproduced by experiments), in less time and with a lower cost compared to experimental techniques.

Actually tools of Computational Fluid-Dynamics (CFD), which is an area of science that study, through numerical simulation, fluid flow, heat transfer, and related transport phenomena, are integrated with others numerical tools, creating a complete design environment, allowing that experiments are done only for final configurations, validations and tests. Many recently researches uses CFD techniques to study AUV problems [4]-[10].

Most existing AUV's uses batteries as power supply for their propulsion systems. However, large drag during the vehicle navigation results in more energy consumption and, consequently, reduction in the vehicle autonomy, which is undesirable by any designer. In this context, this paper aims to evaluate numerically the influence of operation depth, which is operational basic parameter, in the AUV drag coefficient, without and with control surfaces, by CFD. The intention is to obtain data to assist researchers and designers in future projects related to this class of vehicles.

\section{Methodology}

\subsection{The Geometry}

The hull of the AUV studied is torpedo type (cylindrical body with a large ratio between the length and the diameter), due to their good characteristics, like low hydrodynamic drag, good internal volume, simplified access to all equipment and reduction of manufacture cost, making this type of hull the most used by the main commercial manufacturers of this class of vehicles.

To model the profiles of the bow and stern of the vehicle it was used the Myring Equations (Equations (1) and (2)). These theoretical equations describe the shape of the torpedo bodies which generate low drag coefficient [11], and are given as follows:

a) Bow: 


$$
r_{1}\left(x_{1}\right)=\frac{1}{2} D\left[1-\left(\frac{x_{1}-a}{a}\right)^{2}\right]^{1 / n}
$$

b) Stern:

$$
r_{2}\left(x_{2}\right)=\frac{1}{2} D\left[\frac{3 D}{2 c^{2}}-\frac{\operatorname{tg} \theta}{c}\right] x_{2}^{2}+\left[\frac{D}{c^{3}}-\frac{\operatorname{tg} \theta}{c^{2}}\right] x_{2}^{3},
$$

where the parameters of these equations are shown in Figure 1.

In this paper the geometric parameters of AUV's hull considered are shown in Table 1. These parameters were based on dimensions of Afterbodyl hull, studied by [12].

Geometric details of the AUV rudders (NACA0015 profiles) are shown in Figure 2, and are based on [13] and on the Pirajuba AUV studied by [3], which has ratio $L_{t} / D=7.44$, similar the ratio of AUV analyzed in this paper $\left(L_{t} / D=\right.$ 10.00).

For simplification of the analysis, the effect of the propellant over the flow around the AUV was not considered.

\subsection{The Physical Domains and the Numerical Meshes}

For the solution of flow problems using CFD tools is necessary to define continuous fluid domain around the body studied. The continuous domain is then subdivided

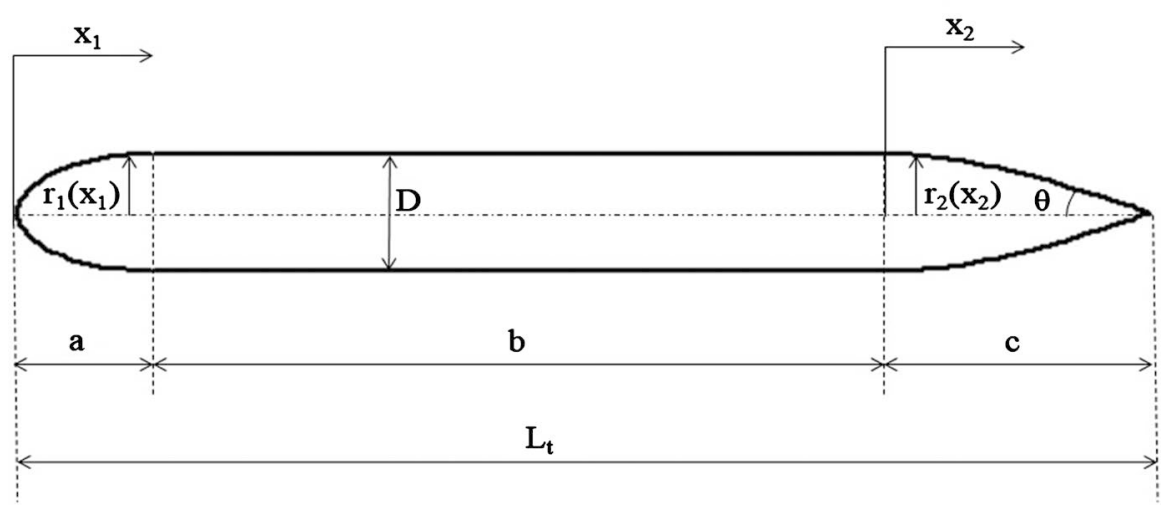

Figure 1. Squematic figure of the AUV hull, with the geometric parameters of Myring Equations.

Table 1. AUV's hull parameters used in the simulations.

\begin{tabular}{cc}
\hline Parameter & Value \\
\hline$L_{t}=a+b+c(\mathrm{~mm})$ & 1400 \\
$a(\mathrm{~mm})$ & 250 \\
$b(\mathrm{~mm})$ & 700 \\
$c(\mathrm{~mm})$ & 450 \\
$D(\mathrm{~mm})$ & 140 \\
$n(-)$ & 2 \\
$\theta\left(^{\circ}\right)$ & 20
\end{tabular}


into small control volumes, becoming it a discrete domain. The set of these control volumes is commonly referred to as the "numerical mesh".

In this paper were create two semi-cylindrical fluid domains (one for AUV with rudders and another for AUV without rudders), aiming to obtain meshes with smaller numbers of control volumes, thus reducing the computational cost. The dimensions of these domains (Figure 3) were based in the work of [5], that studied the flow around AUV hull (torpedo type) with $L_{t} D=9.00$, and are shown in Table 2.

For generation of the geometries and numerical meshes that represent the studied domains was used the commercial software ICEM-CFD 15.0.

Initially it was procedure the mesh convergence study, aiming to obtaining independence of simulations results, due to the increment of mesh elements. The numerical meshes used are hybrids (tetrahedral and prisms control volumes), with 454,950 elements (medium $y^{+}=11$ ) for the situation of AUV without rudders
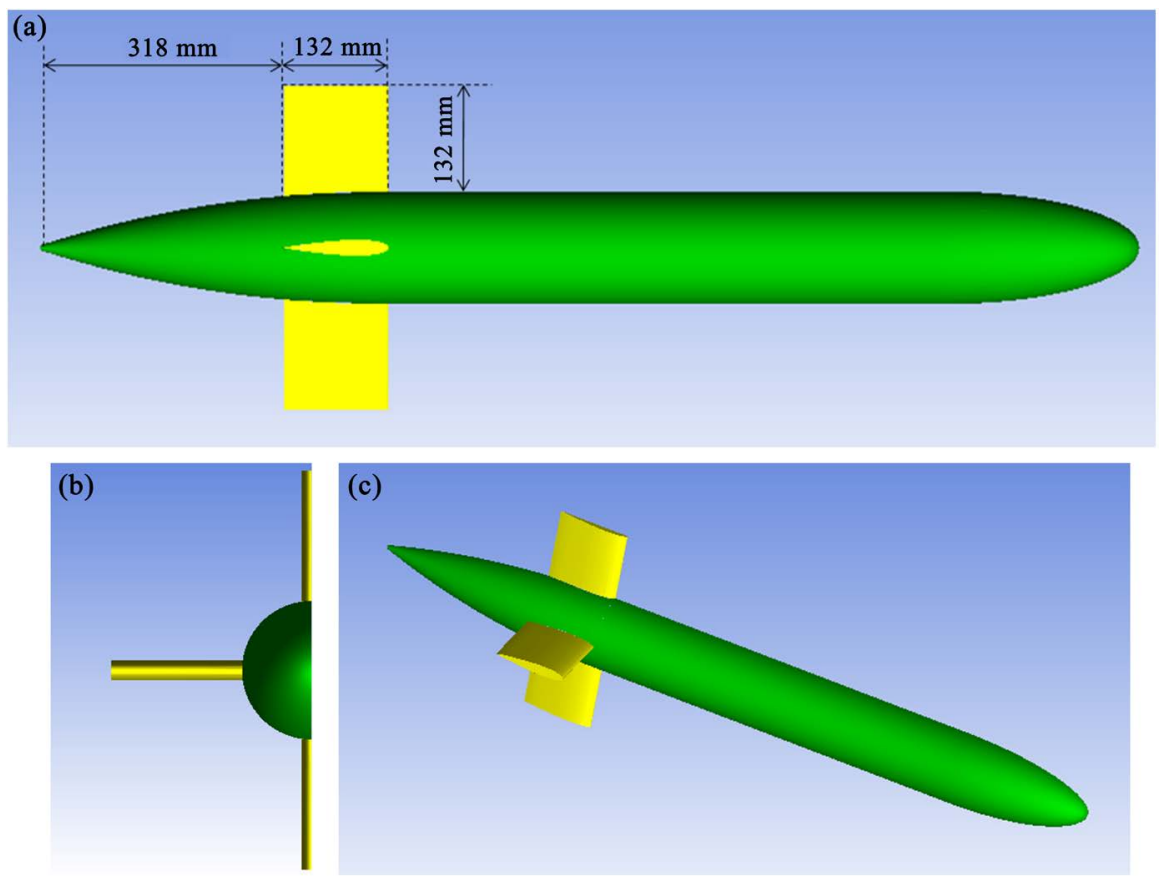

Figure 2. Geometric details of the AUV studied in lateral (a), frontal (b) and isometric (c) views.

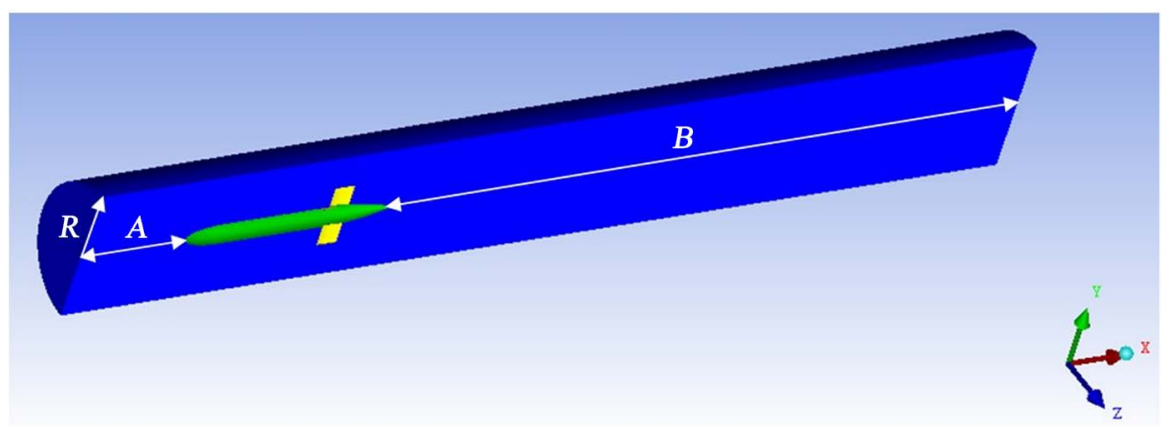

Figure 3. Fluid domain studied. 
(mesh 1) and 1,012,855 elements (medium $y^{+}=8$ ) for the situation of AUV with rudders (mesh 2), and are showed in Figure 4. The refinement of both meshes are similar, but mesh 2 has more than twice the numbers of elements of mesh 1 due to rudders (27\% additional surface area), which require a large quantity of additional prismatic elements near these control surfaces.

$y^{+}$is a dimensionless distance that is a relevant parameter in the modeling of external flow. This parameter, which is commonly used to define the ideal mesh refinement in the wall regions, is calculated by the following equation:

$$
y^{+}=\frac{\Delta n}{\mu / \sqrt{\rho \tau_{w}}},
$$

where $\Delta n$ is the height of the first layer of control volumes, measured vertically to the wall, $\mu$ is the fluid dynamic viscosity, $\rho$ is the fluid density and $\tau_{w}$ is the shear stress in the wall.

Table 2. Geometric parameters that define the dimensions of fluid domain studied.

\begin{tabular}{cc}
\hline Parameter & Value \\
\hline$A(\mathrm{~mm})$ & $0.5 L_{t}=700$ \\
$B(\mathrm{~mm})$ & $3 L_{t}=5200$ \\
$R(\mathrm{~mm})$ & $3 D=420$ \\
\hline
\end{tabular}

(a)

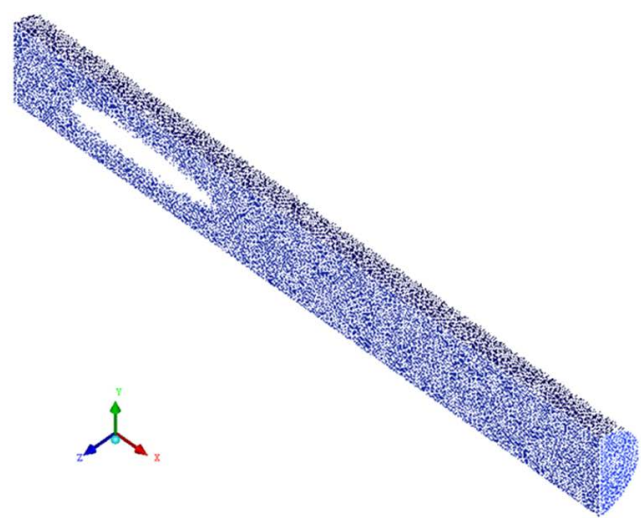

(b)

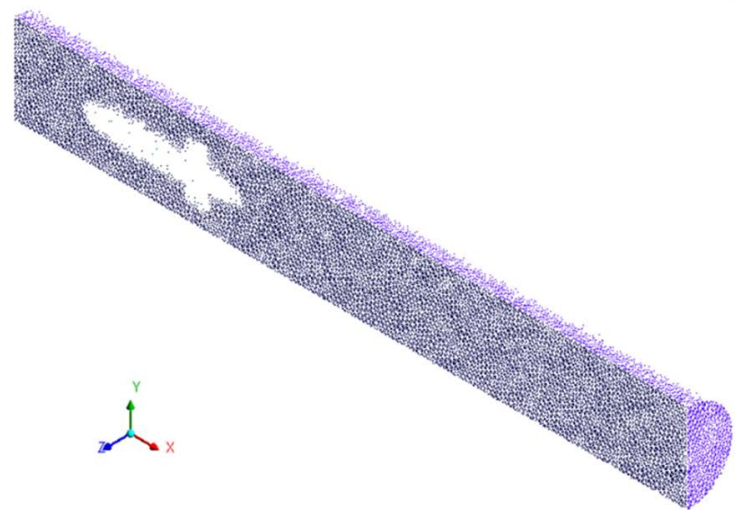

Figure 4. Meshes created in the studied domain, for situations of AUV without (a) and with (b) rudders (meshes 1 and 2, respectively). 
Much care must be taken when constructing numerical meshes aimed at solving problems of flow around immersed bodies. Here, many factors were taken into account [14]. It was used an equation that provides the average thickness of the kinetic boundary layer (region where viscous flow effects are important) around immersed bodies, $\delta$, as follows:

$$
\delta=0.035 L_{t} R e^{-1 / 7},
$$

where $R e$ is Reynolds number of the flow, calculated as follow:

$$
R e=\frac{\rho|\boldsymbol{U}| L_{t}}{\mu}
$$

where $|\boldsymbol{U}|$ is the norm of flow velocity vector and $\mu$ is the fluid dynamic viscosity. The meshes were built with great refinement near the surface of the AUV (boundary layer region), in order to encompass precisely the viscous effects of the flow around the AUV.

The Reynolds number can be defined in volumetric basis, as follows:

$$
\operatorname{Re}_{V}=\frac{\rho|\boldsymbol{U}| V^{1 / 3}}{\mu}
$$

where $R e_{V}$ is the volumetric Reynolds number of the flow, and $V$ is the volume of AUV hull.

Figure 5 shows details of the mesh 2 around the region of bow, upper rudder and stern, respectively.

\subsection{Mathematical Modeling}

To investigate the single-phase flow around the AUV, it was considered a three-dimensional, steady-state, incompressible, isothermal and turbulent flow.

The general equations used in this work are:

a) Mass conservation equation:

$$
\frac{\partial \rho}{\partial t}+\nabla \cdot(\rho \boldsymbol{U})=0
$$

b) Momentum conservation equation:

$$
\frac{\partial(\rho \boldsymbol{U})}{\partial t}+\nabla \cdot(\rho \boldsymbol{U} \otimes \boldsymbol{U})-\nabla \cdot\left(\mu_{e f f} \nabla \boldsymbol{U}\right)=-\nabla p^{\prime}+\nabla \cdot\left(\mu_{e f f} \nabla \boldsymbol{U}\right)^{\mathrm{T}}+\rho \boldsymbol{g},
$$

where $p^{\prime}$ is the corrected pressure, which depends of turbulent model to be used, $\boldsymbol{g}$ is the local gravity acceleration vector (adopted value $9.81 \mathrm{~m} / \mathrm{s}^{2}$ ) and $\mu_{\text {eff }}$ is the effective viscosity, calculated as follows:

$$
\mu_{\text {eff }}=\mu+\mu_{t}
$$

where $\mu_{t}$ is the turbulent viscosity.

c) Turbulence model equations:

It is necessary to add in the model new equations to predict the phenomenon of turbulence that is present in the flow. The turbulence consists of fluctuations in the flow field in time and space (time-dependent velocity and pressure fields). 


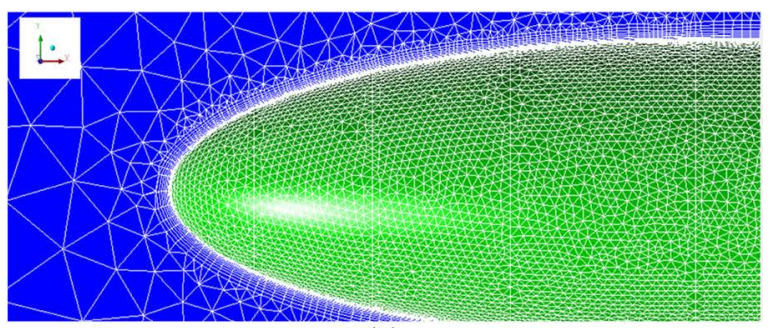

(a)

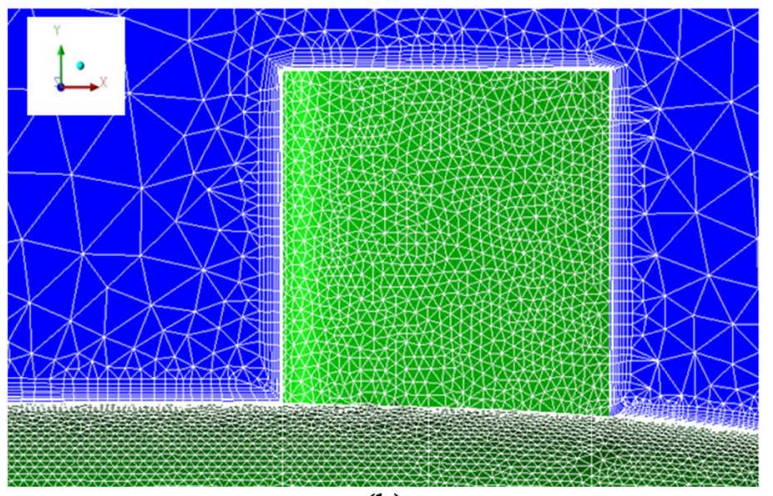

(b)

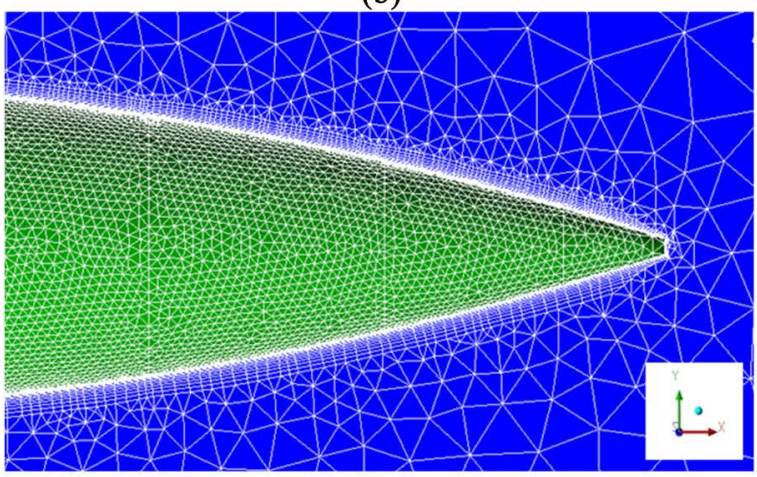

(c)

Figure 5. Details of mesh 2 around the regions of bow (a), upper rudder (b) and stern (c).

It is a complex process and can have a significant effect on the flow characteristics. Turbulence occurs when the inertial forces acting on the fluid becomes significantly higher than the viscous forces and is characterized by a high Reynolds Number of the flow. Turbulence can also be caused by surface roughness, which induces secondary flow (i.e. vortices) [15].

The turbulence model adopted in this work is the Shear Stress Transport model (SST model), with the kinetic boundary layer fully turbulent. This model is based on the $k-\omega$ turbulence model. This model was used because of its good treatment of external flow with high Reynolds numbers.

The SST model introduces two new variables in the problem, which is the turbulent kinetic energy $(k)$ and the turbulent frequency $(\omega)$. These variables are calculated, respectively, by:

$$
\frac{\partial(\rho k)}{\partial t}+\nabla \cdot(\rho \boldsymbol{U} k)=\nabla \cdot\left[\left(\mu+\frac{\mu_{t}}{C_{k 1}}\right) \nabla k\right]+P_{k}-C_{k 2} \rho k \omega,
$$


where $P_{k}$ is the turbulence production, $C_{k 1}=2.000$ and $C_{k 2}=0.090$. And:

$$
\frac{\partial(\rho \omega)}{\partial t}+\nabla \cdot(\rho \boldsymbol{U} \omega)=\nabla \cdot\left[\left(\mu+\frac{\mu_{t}}{C_{\omega 1}}\right) \nabla \omega\right]+C_{\omega 2} P_{k} \frac{\omega}{k}-C_{\omega 3} \rho \omega^{2},
$$

where $C_{\omega 1}=2.000, C_{\omega 2}=0.556$ and $C_{\omega 3}=0.075$. In the $S S T$ model the parameters $\mu_{t} p^{\prime}$ and $P_{k}$ are given as follows:

$$
\begin{gathered}
\mu_{t}=\rho \frac{k}{\omega}, \\
p^{\prime}=p_{d}+\frac{2}{3} \rho k \text { and } \\
P_{k}=\mu_{t} \nabla \boldsymbol{U} \cdot\left(\nabla \boldsymbol{U}+\nabla \boldsymbol{U}^{\mathrm{T}}\right)-\frac{2}{3} \nabla \cdot \boldsymbol{U}\left(3 \mu_{t} \nabla \cdot \boldsymbol{U}+\rho k\right),
\end{gathered}
$$

where $p_{d}$ is the dynamic pressure, calculated by the equation:

$$
p_{d}=\frac{1}{2} \rho|\boldsymbol{U}|^{2} .
$$

Total pressure, $p_{p}$ is calculated by:

$$
p_{t}=p_{s}+p_{d},
$$

where $p_{s}$ is static pressure.

According to [16], the action of viscosity is diminish the fluid velocity past a surface and, thus, decreasing the fluid momentum within the boundary layer. It strongly affects the overall flow behavior past the surface. Since, fluid flow is governed by the pressure distribution on the boundary layer. We must analyze both the retarding action of viscosity and the imposed pressure distribution. Thus, the drag coefficient in the flow was determined. The total drag force is generated by the friction and pressure forces acting on a body immersed in a flowing fluid.

The friction drag is due to the boundary layer surface shear stress, while pressure drag is due to pressure difference in the flow direction resulting from formation of the wake in the downstream region.

The drag on a body is usually expressed in terms of a dimensionless drag coefficient. The total drag coefficient in volumetric basis is calculated by:

$$
C_{d v}=C_{d p v}+C_{d v v}=\frac{F_{d p}}{\frac{1}{2} \rho U_{*}^{2} V^{2 / 3}}+\frac{F_{d f}}{\frac{1}{2} \rho U_{*}^{2} V^{2 / 3}},
$$

where $C_{d v}$ is the volumetric drag coefficient, $C_{d p v}$ is the volumetric pressure drag coefficient, $C_{d f v}$ is the volumetric friction drag coefficient, $F_{d p}$ is the pressure drag force, $F_{d f}$ is the friction drag force and $U_{*}$ is the free stream parallel fluid velocity. The parameters $F_{d p}$ and $F_{d f}$ are calculated by:

$$
\begin{gathered}
F_{d p}=\left(\int_{A} p_{d} \mathrm{~d} A\right) \hat{i}, \\
F_{d f}=\left(\int_{A} \tau_{w} \mathrm{~d} A\right) \hat{i},
\end{gathered}
$$


where $A$ is the AUV superficial area and $\hat{i}$ is the unit vector in the parallel flow direction.

\subsection{Boundary Conditions and Fluids Properties}

Figure 6 shows the domain with indicative information related to boundary conditions and Table 3 shows specified values of these boundary conditions.

The fluid adopted in the validations simulations was pure water. The physical properties of this fluid are shown in Table 4.

The fluid adopted in the simulations that evaluates the influence of operation depth in drag coefficient was sea water with $35 \mathrm{~g} / \mathrm{L}$ of salinity. The physical properties of this fluid, for equatorial and tropical areas, are shown in Table 5.

Table 6 shows the considerations adopted for the numerical solver.

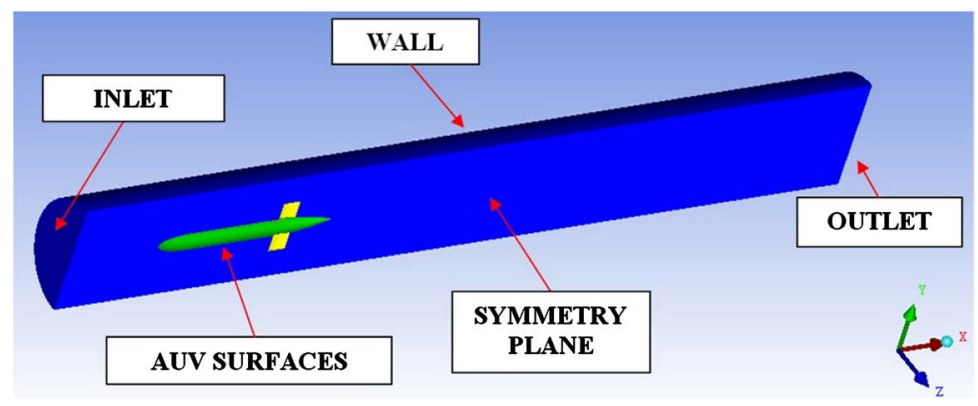

Figure 6. Physical specifications of the domain with the boundary conditions.

Table 3. Boundary conditions used in the simulations.

\begin{tabular}{|c|c|c|}
\hline Boundary & Condition & Value \\
\hline Inlet & Prescribed velocity & $0.4 \hat{i}-1.4 \hat{i} \mathrm{~m} / \mathrm{s}$ \\
\hline Wall & Prescribed pressure ${ }^{3}$ & $5.1-10.2 \mathrm{MPa}$ \\
\hline AUV surfaces ${ }^{1}$ & Prescribed velocity & $0 \mathrm{~m} / \mathrm{s}$ \\
\hline Outlet $^{2}$ & Prescribed pressure ${ }^{3}$ & $5.1-10.2 \mathrm{MPa}$ \\
\hline Symmetry plane & Symmetry & - \\
\hline
\end{tabular}

${ }^{1}$ No slip and smooth wall; ${ }^{2}$ Opening condition; ${ }^{3}$ On the operation depth in the water.

Table 4. Physical properties of the pure water.

\begin{tabular}{cc}
\hline Propertie & Value \\
\hline Density $\left(\mathrm{kg} / \mathrm{m}^{3}\right)$ & 997 \\
Dynamic viscosity $(\mathrm{mPa} \cdot \mathrm{s})$ & 0.8899 \\
\hline
\end{tabular}

Table 5. Physical properties of the sea water.

\begin{tabular}{cccc}
\hline Depth $(\mathrm{m})$ & Temperature $\left({ }^{\circ} \mathrm{C}\right)$ & Density $\left(\mathrm{kg} / \mathrm{m}^{3}\right)$ & Dynamic viscosity $(\mathrm{mPa} \cdot \mathrm{s})$ \\
\hline 500 & 25 & 1023 & 0.9600 \\
750 & 15 & 1026 & 1.2300 \\
1000 & 3 & 1028 & 1.7200 \\
\hline
\end{tabular}


In order to predict the drag coefficients at different situations, it was proposed the following linear model for these parameters as a function of the operation depth $(Y)$ :

$$
C=\bar{A}+\bar{B} \cdot Y
$$

where $\bar{A}$ and $\bar{B}$ are coefficients of the equation and $C$ is the dependent variable $\left(C_{d v}, C_{d p v}\right.$ or $\left.C_{d f v}\right)$

This model was fitted by numerical simulations and the least square error technique. For all of the simulations was used the commercial software ANSYS-CFX 15.0, which makes use of the finite volumes method based on finite elements method to solve the problem under study.

Equation (20) is valid in the interval $500 \leq Y(\mathrm{~m}) \leq 1000$.

\section{Results and Discussion}

\subsection{AUV without Rudders (Validation)}

To validate the methodology for constructing the numerical meshes and mathematical model used in this study, was done comparison between the predicted volumetric drag coefficient and the obtained experimentally by [12], that studied similar AUV without rudders.

Table 7 and Figure 7 show the comparison between the numerical and experimental volumetric drag coefficients versus volumetric Reynolds number for

Table 6. Considerations adopted in numerical solver.

\begin{tabular}{cc}
\hline Parameter & Consideration \\
\hline Primary convergence criterion & Root Main Square (RMS) equations error $<10^{-5}$ \\
Secondary convergence criterion & Reached 300 iterations, with convergence of $C_{d v}$ \\
Advection scheme & High resolution \\
Interpolation scheme for pressure & Trilinear \\
Interpolation scheme for velocity & Trilinear \\
\hline
\end{tabular}

Table 7. Comparison between experimental and numerical results of the volumetric drag coefficients for flow around the AUV without rudders.

\begin{tabular}{|c|c|c|c|c|c|}
\hline$U_{*}(\mathrm{~m} / \mathrm{s})$ & $\begin{array}{c}\operatorname{Re} \times 10^{-5} \\
(-)\end{array}$ & $\begin{array}{c}R e_{V} \times 10^{-5} \\
(-)\end{array}$ & $\begin{array}{c}\text { Experimental } \\
C_{d_{v}}{ }^{1}(-)\end{array}$ & $\begin{array}{l}\text { Numerical } \\
\qquad C_{d_{V}}(-)\end{array}$ & $\begin{array}{c}\text { Difference between } \\
\text { numerical and } \\
\text { experimental } C_{d v}^{2}(\%)\end{array}$ \\
\hline 0.4 & 6.27 & 1.05 & 0.0489 & 0.0460 & 5.9 \\
\hline 0.6 & 9.41 & 1.57 & 0.0451 & 0.0424 & 6.0 \\
\hline 0.8 & 12.55 & 2.10 & 0.0434 & 0.0401 & 7.6 \\
\hline 1.0 & 15.68 & 2.62 & 0.0419 & 0.0385 & 8.1 \\
\hline 1.2 & 18.82 & 3.15 & 0.0407 & 0.0372 & 8.5 \\
\hline 1.4 & 21.96 & 3.67 & 0.0389 & 0.0362 & 6.8 \\
\hline
\end{tabular}


AUV without rudders. From the analysis of the results we can note that the maximum and the average difference between the predicted and experimental volumetric drag coefficients were small, $8.1 \%$ and $7.2 \%$, respectively. These deviations can be attributed to the association of experimental errors, numerical errors and the small variation between the computationally simulated hull geometry and that used in the experiments. The low deviations between the results validate the methodology used in this work, showing that it describe well the studied physical phenomenon.

\subsection{AUV without and with Rudders (Hydrodynamic Analysis)}

Figures 8-10 show the comparison between results of volumetric drag coefficients, volumetric pressure drag coefficient and volumetric friction drag coefficient, respectively, versus operating depth obtained for AUV without (Table 8)

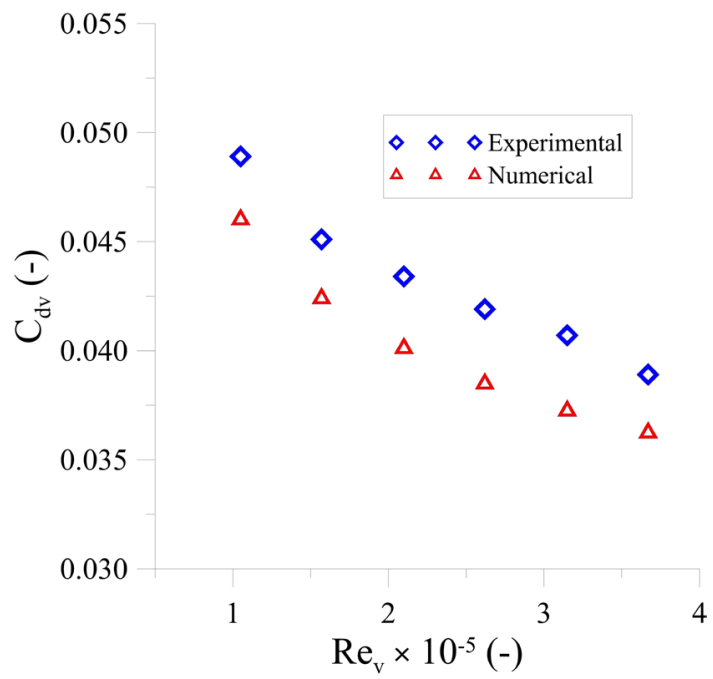

Figure 7. Volumetric drag coefficients versus volumetric Reynolds number for AUV without rudders.

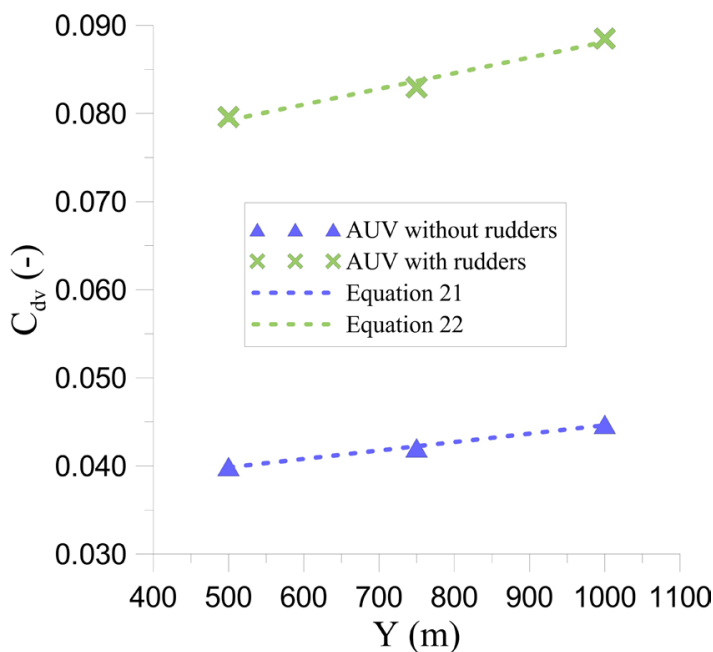

Figure 8. Volumetric drag coefficient versus operating depth obtained for AUV without and with rudders. 


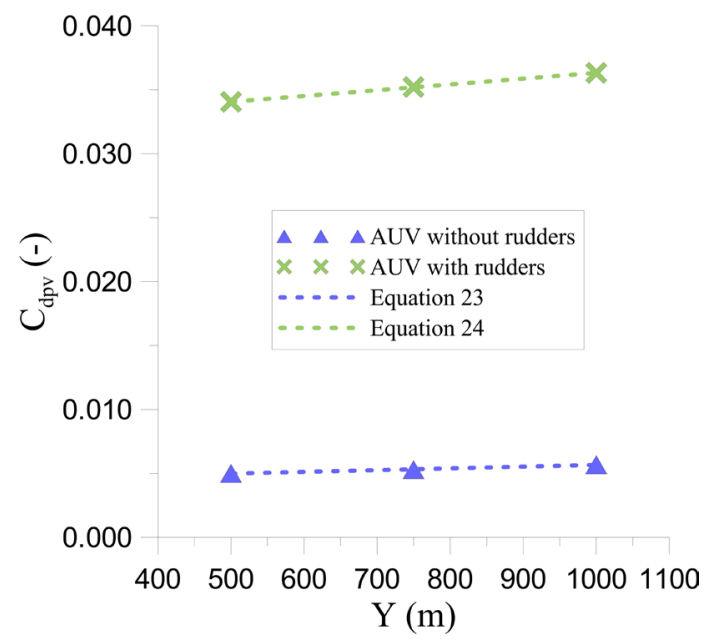

Figure 9. Volumetric pressure drag coefficient versus operating depth obtained for AUV without and with rudders.

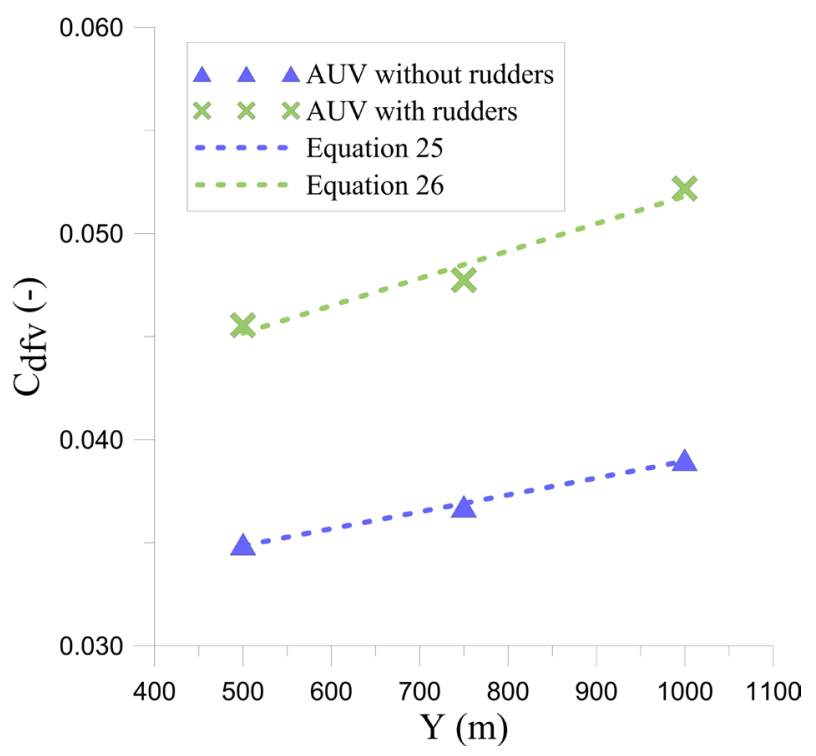

Figure 10. Volumetric friction drag coefficient versus operating depth obtained for AUV without and with rudders.

Table 8. Volumetric drag coefficient, volumetric pressure drag coefficient and volumetric friction drag coefficient versus operating depth obtained for AUV without rudders.

\begin{tabular}{cccccccc}
\hline$Y(\mathrm{~m})$ & $\begin{array}{c}R e \times 10^{-5} \\
(-)\end{array}$ & $\begin{array}{c}R e_{V} \times 10^{-5} \\
(-)\end{array}$ & $C_{d v}(-)$ & $C_{d p v}(-)$ & $C_{d p v} / C_{d v}(\%)$ & $C_{d f v}(-)$ & $C_{d f v} / C_{d V}(\%)$ \\
\hline 500 & 14.92 & 2.71 & 0.0399 & 0.0050 & 12.5 & 0.0349 & 87.5 \\
750 & 11.68 & 2.12 & 0.0421 & 0.0053 & 12.6 & 0.0368 & 87.4 \\
1000 & 8.37 & 1.52 & 0.0447 & 0.0057 & 12.8 & 0.0390 & 87.2 \\
\hline
\end{tabular}

and with (Table 9) rudders, and free stream fluid velocity equal to $1.0 \mathrm{~m} / \mathrm{s}$.

From analysis of these results it can be verified a linearly increasing behavior of the volumetric drag coefficient with the increase in the operation depth for both situations, AUV without and with rudders. This phenomenon can be explained, 
Table 9. Volumetric drag coefficient, volumetric pressure drag coefficient and volumetric friction drag coefficient versus operating depth obtained for AUV with rudders.

\begin{tabular}{cccccccc}
\hline$Y(\mathrm{~m})$ & $\begin{array}{c}R e \times 10^{-5} \\
(-)\end{array}$ & $\begin{array}{c}R e_{V} \times 10^{-5} \\
(-)\end{array}$ & $C_{d v}(-)$ & $C_{d p v}(-)$ & $C_{d p v} / C_{d v}(\%)$ & $C_{d f v}(-)$ & $C_{d f v} / C_{d v}(\%)$ \\
\hline 500 & 14.92 & 2.71 & 0.0796 & 0.0341 & 42.8 & 0.0455 & 57.2 \\
750 & 11.68 & 2.12 & 0.0830 & 0.0352 & 42.4 & 0.0477 & 57.6 \\
1000 & 8.37 & 1.52 & 0.0885 & 0.0363 & 41.0 & 0.0522 & 59.0 \\
\hline
\end{tabular}

basically, by the significant increase in sea water viscosity due to the considerable drop in temperature as AUV moves deeper into the ocean layers.

Further, it was observed that the average volumetric drag coefficient of AUV with rudders is approximately twice when compared with the situation of AUV without rudders, showing that the use of control surfaces has a strong impact on the total drag of the vehicle, for the simulated conditions. It was also verified an almost constant value of the volumetric pressure drag coefficient with the increase in the operation depth, for the AUV in both situations (without and with rudders), showing that the change in the operational depth parameter has little influence on the pressure drag of the vehicle. In the case of AUV with rudders it was observed a volumetric pressure drag coefficient almost 7 times higher than the situation of AUV without rudders, due to the $68 \%$ increase in the frontal area of the vehicle. This additional part in the vehicle generates an increase in the flow separation and consequent increase in the wake region. Furthermore, it was possible to verify that the average percentage of pressure drag in relation to the total drag of the vehicle increased from $12.6 \%$ to $42.4 \%$, considering the AUV without and with rudders, respectively. As reported in the methodology, based on the simulations results it were obtained correlations between the parameters $C_{d w} C_{d p v}$ and $C_{d f v}$ as a function of the operation depth, $Y$.

It was also found that volumetric friction drag coefficient for the AUV with rudders is approximately $30 \%$ higher than the obtained with the AUV without the rudders, due to the $29 \%$ increase in vehicle wetted area, thus increasing the area subjected to viscous shear stress generated by the fluid flow. In addition, it was possible to verify that the average percentage of frictional drag in relation to the total drag of the vehicle dropped from $87.4 \%$ to $57.6 \%$, considering AUV with and without rudders, respectively.

In Table 10 are shown the statistical parameters obtained with the linear regression of the Equation (20) to predicted values of the drag coefficients.

Based on the high determination coefficient reported in Table $10\left(R^{2}>0.96\right)$, we can verify that a good agreement was obtained. Thus, we state that all drag coefficients present linear behavior with operation depth, in both AUV design.

Figure 11 and Figure 12 show the total pressure fields around the AUV without and with rudders, respectively, in the longitudinal symmetry plane, for operation depth $750 \mathrm{~m}$. From the analysis of these figures it is verified the higher pressure in the stagnation point of the flow, in the bow, for both situations. 
Table 10. Statistical parameters obtained by Equation (20).

\begin{tabular}{cccccc}
\hline AUV & Equation & $\begin{array}{c}\text { Dependent } \\
\text { variable }(-)\end{array}$ & $\begin{array}{c}\text { Coefficient } \\
\bar{A}(-)\end{array}$ & $\begin{array}{c}\text { Coefficient } \bar{B} \times \\
10^{4}\left(\mathrm{~m}^{-1}\right)\end{array}$ & $R^{2}(-)$ \\
\hline \multirow{3}{*}{ Without } & 21 & $C_{d v}$ & 0.0351 & 0.0956 & 0.9961 \\
rudders & 23 & $C_{d p v}$ & 0.0043 & 0.0137 & 0.9951 \\
& 25 & $C_{d f v}$ & 0.0308 & 0.0820 & 0.9963 \\
\hline \multirow{2}{*}{$\begin{array}{c}\text { With } \\
\text { rudders }\end{array}$} & 22 & $C_{d v}$ & 0.0703 & 0.0178 & 0.9802 \\
& 24 & $C_{d p v}$ & 0.0318 & 0.0452 & 0.9998 \\
\hline
\end{tabular}

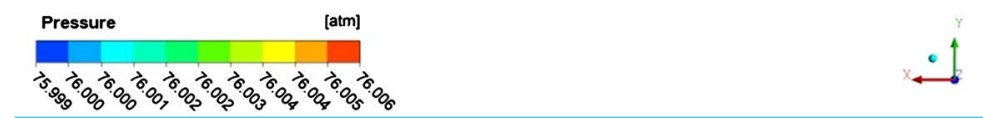

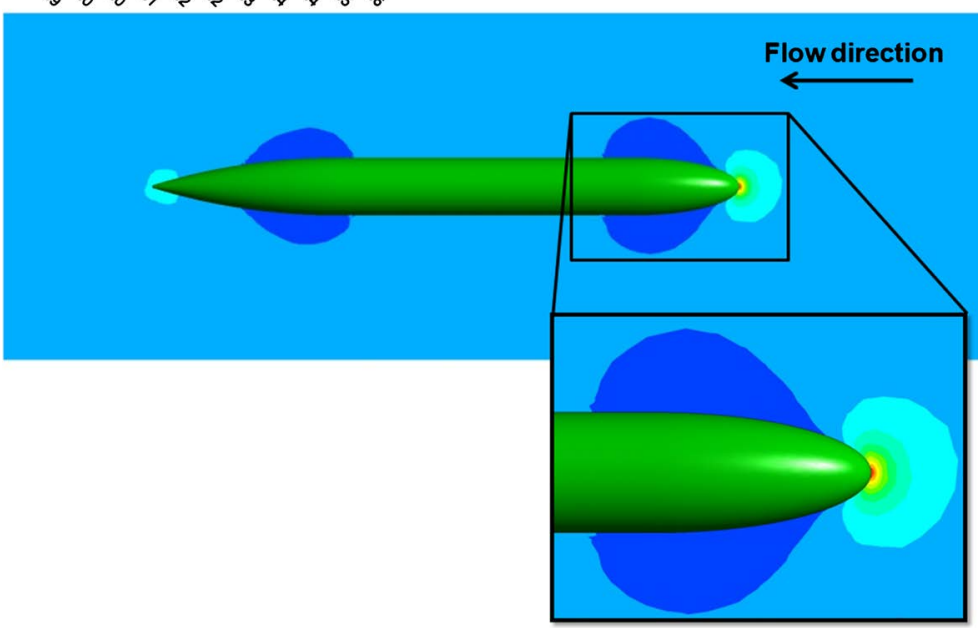

Figure 11. Pressure field around the AUV without rudders.

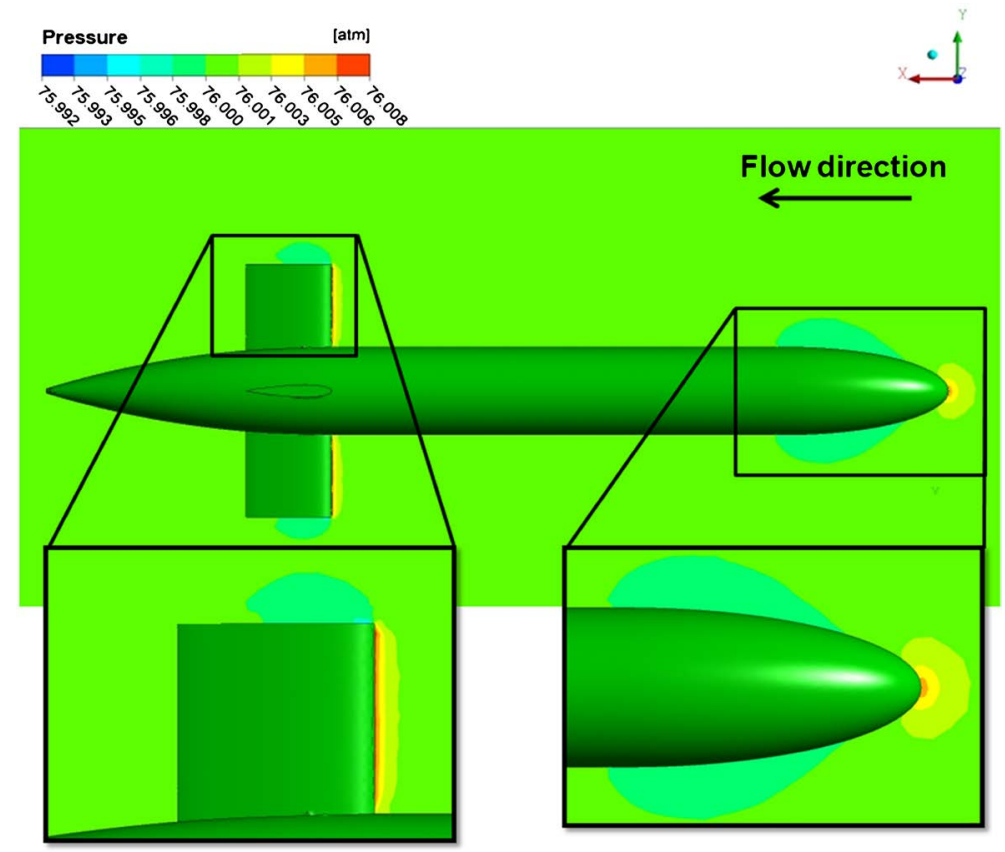

Figure 12. Pressure field around the AUV with rudders. 
Further, it is verified high pressure values and low range of this parameter. This is due the high contribution of static pressure, $p_{s}$, in the total pressure, due the big depth in that the study was performed. For the case of AUV with rudders there are additional high pressure zones at the leading edges of the rudders, as well as additional low pressure zones at the rudder tips. The same pressure behavior was verified for simulations in operation depths of 500 and $1000 \mathrm{~m}$.

Figure 13 shows the total pressure field around the AUV with rudders in the longitudinal plane located $0.15 \mathrm{~m}$ from the AUV centerline, for operation depth $750 \mathrm{~m}$. This plane cross one of the rudders of the vehicle. From the analysis of this figure we can see that there is a high pressure zone on the leading edge of this rudder, as well as symmetrical low pressure zones on both sides. This physical situation is expected, since the NACA0015 profile is symmetrical and the AUV moves at null attack angle. The same behavior was verified for simulations in operation depths of 500 and $1000 \mathrm{~m}$.

Figure 14 and Figure 15 illustrate the velocity fields around the AUV without and with rudders, respectively, in the longitudinal symmetry plane, for operation depth $750 \mathrm{~m}$. From the analysis of these figures it was verified low speed zones at the bow of the vehicle and a wake region in the stern, for both situations. The tip of the AUV bow is a stagnation point, where we can verify null velocity and maximum pressure. For the case of AUV with rudders, it is also found that the flow around the vehicle is considerably more disturbed, especially downstream of the rudders. The same behavior was verified for simulations in operation depths of 500 and $1000 \mathrm{~m}$.

Figure 16 shows the velocity field around the AUV with rudders in the longitudinal plane located $0.15 \mathrm{~m}$ from the AUV centerline, for operation depth 750 $\mathrm{m}$. This plane cross one of the rudders of the vehicle. From the analysis of this

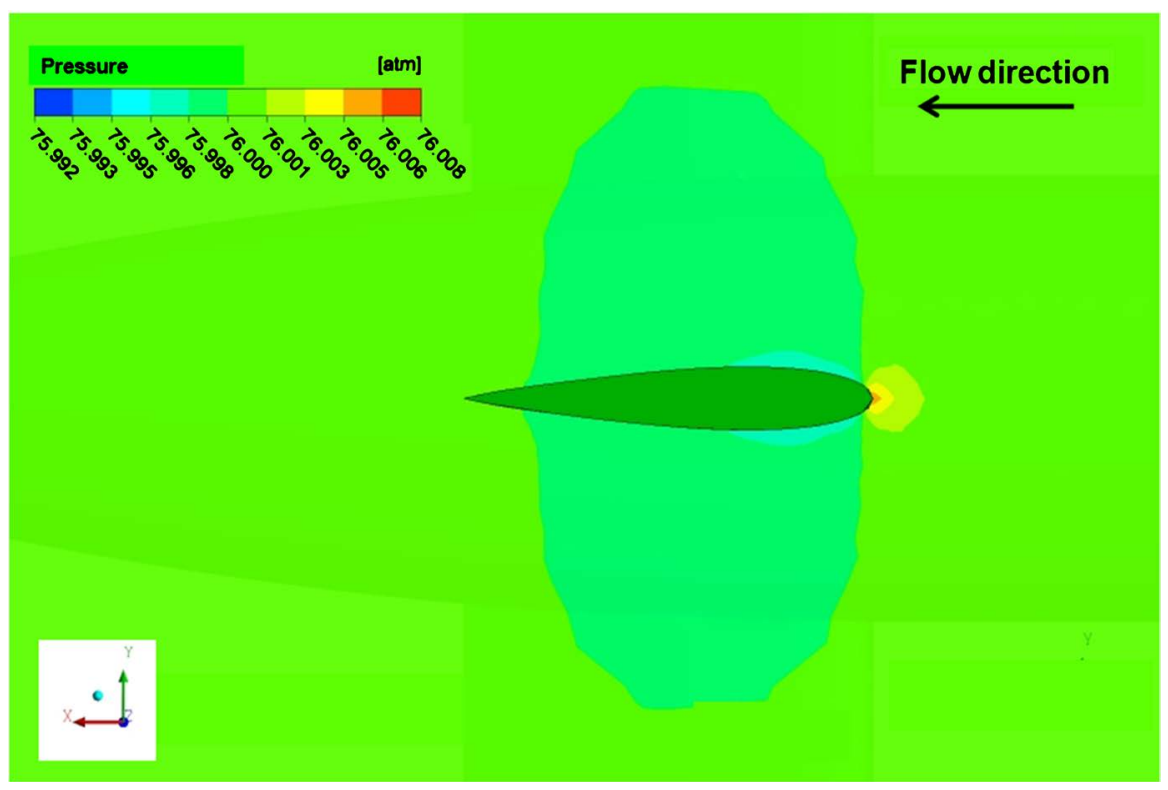

Figure 13. Pressure field around the AUV, in a plane that cross one of the rudders of the vehicle. 
figure we can see that there are low velocities zones on the leading edge and in the wake region of this rudder, as well as symmetrical high velocities zones on their both sides, a physically expected fact, due the characteristics already mentioned of NACA0015 profile and the null attack angle of vehicle. Similar behavior was verified for simulations in operation depths of 500 and $1000 \mathrm{~m}$.

Figure 17 and Figure 18 show the velocity fields (streamlines) around the AUV with rudders in the longitudinal symmetry plane and in the longitudinal

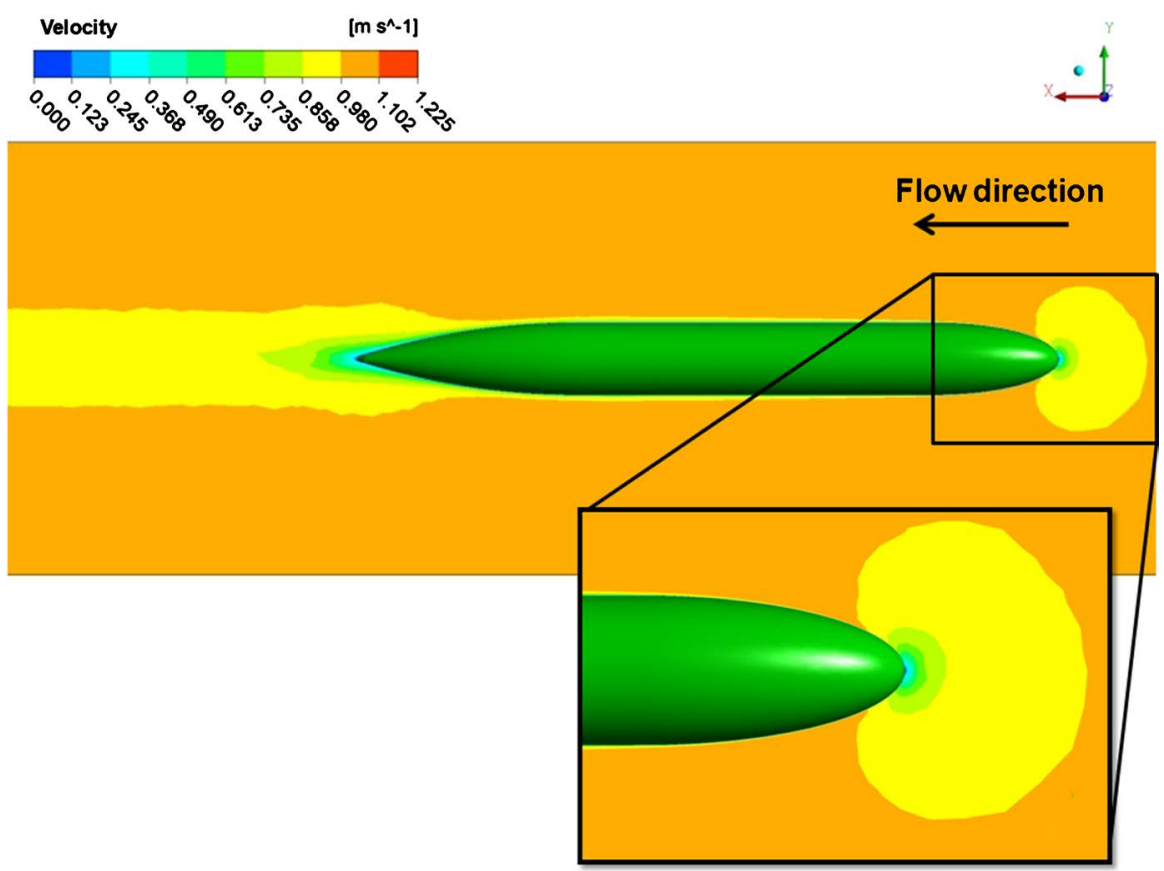

Figure 14. Velocity field around the AUV without rudders.

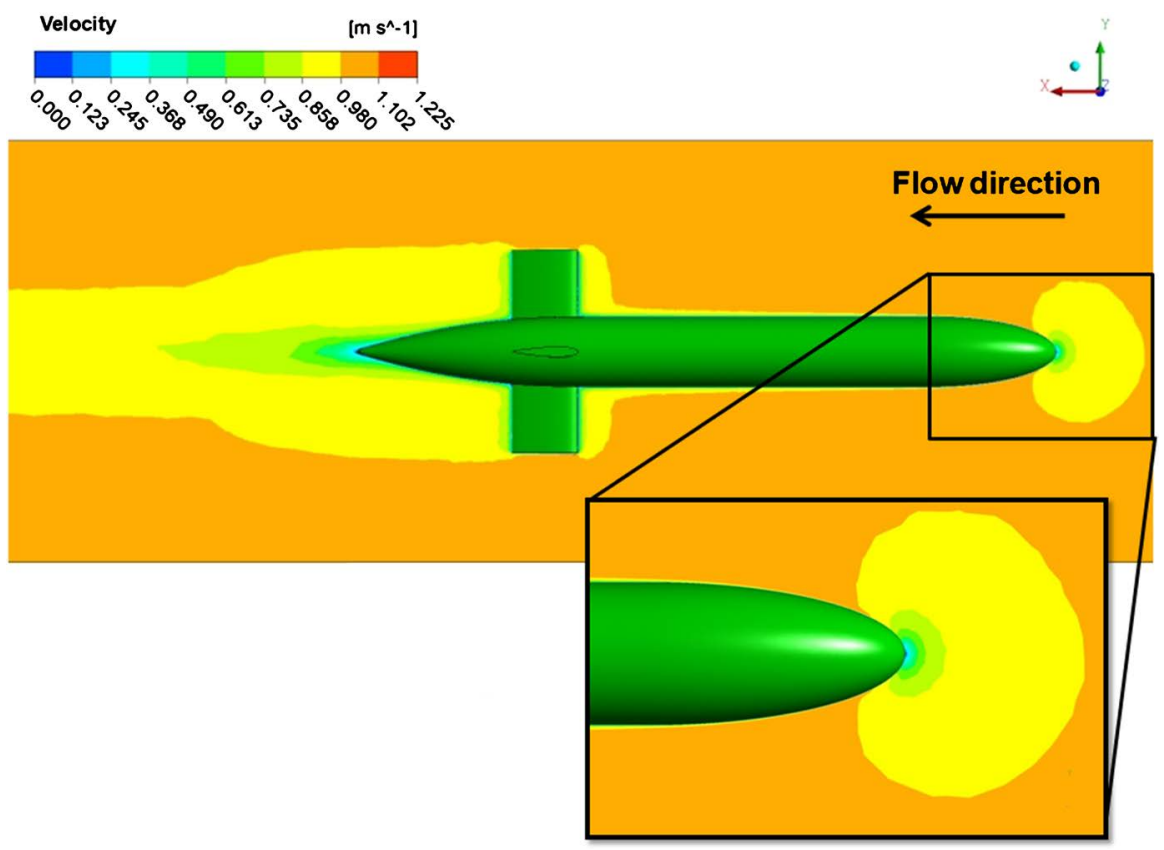

Figure 15. Velocity field around the AUV with rudders. 


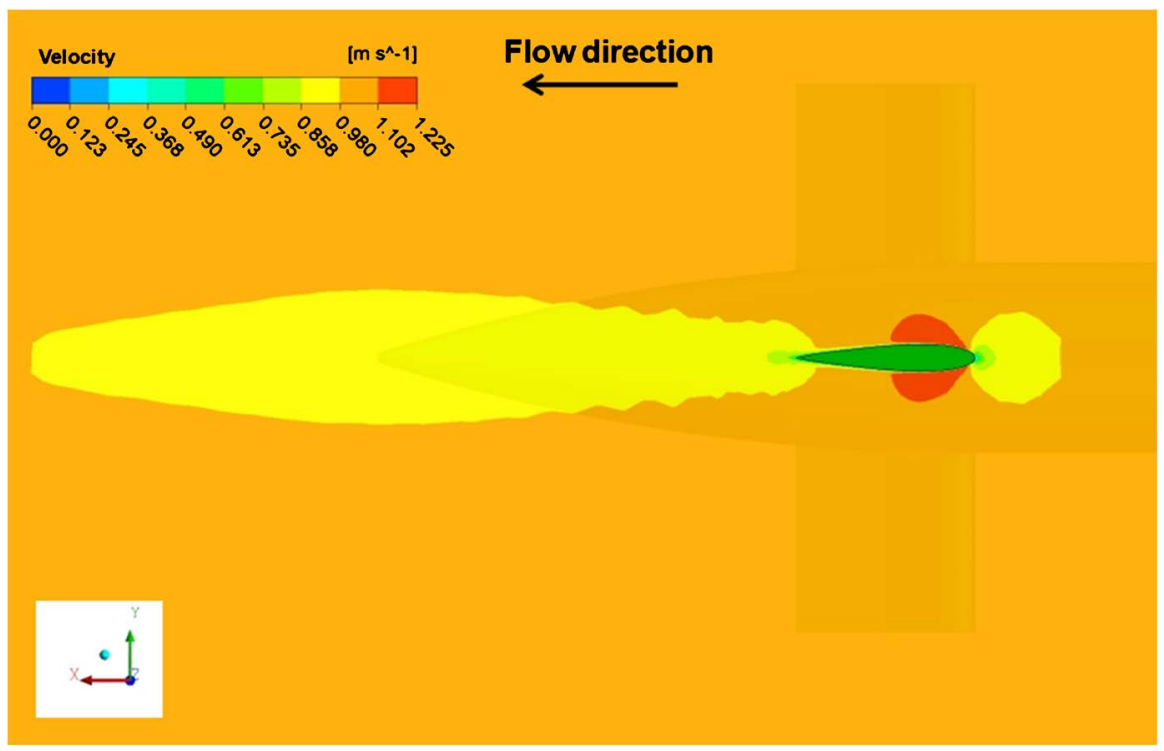

Figure 16. Velocity field around the AUV, in a plane that cross one of the rudders of the vehicle.

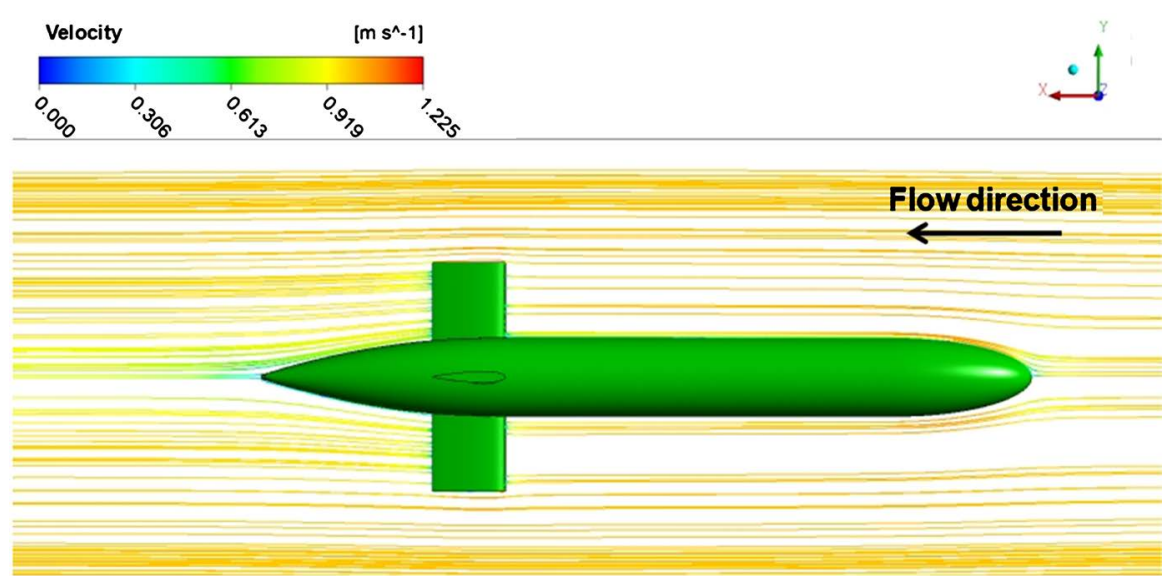

Figure 17. Streamlines showing the velocity field around the AUV with rudders.

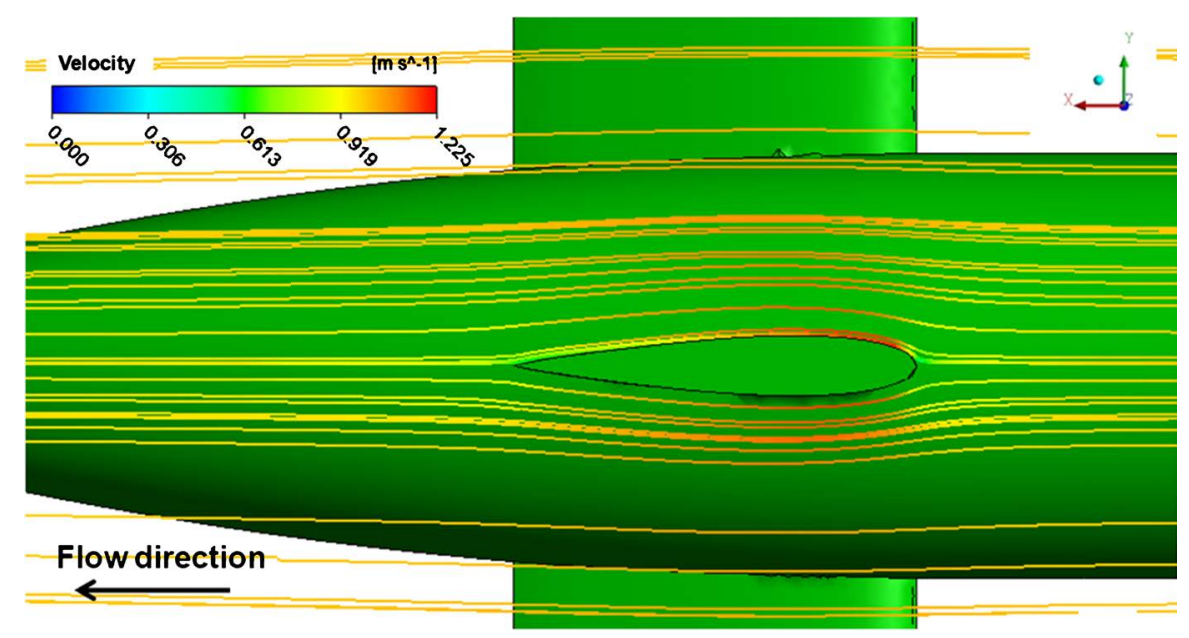

Figure 18. Streamlines showing the velocity field around the horizontal rudder of the AUV. 
plane located $0.15 \mathrm{~m}$ from the AUV centerline (crossing one of the rudders), respectively, for operation depth $750 \mathrm{~m}$. From the analysis of these figures we can verify that the streamlines were quite ordered around the AUV, which is due to the good hydrodynamic geometry of the hull and rudders of the vehicle. The same pattern was verified for simulations in operation depths of 500 and $1000 \mathrm{~m}$.

\section{Conclusions}

This paper study the fluid flow behavior past over AUV's, without and with control surfaces (rudders), by Computational Fluid-Dynamics (CFD). Results of the drag coefficient, pressure, velocity and streamlines distribution around the vehicles were presented and analyzed.

Based on the results it can be concluded that the numerical-mathematical model used in this paper represented well the phenomenon of the flow around the AUV studied and that the methodology used was satisfactory. It was also observed that the average total drag of the AUV with rudders was about twice higher than the AUV without rudders, and the contribution due to pressure and friction effects are practically constant and increasing, respectively, when the operation depth is increased, for both situations, with and without rudders. Additionally, it was obtained a linear model to predict the drag coefficients as a function of the operation depth. Finally, the study has proved that CFD tools have strong relevance in the development and improvement of naval projects, being essential in the current scenario of technological advances and cost reduction.

\section{Acknowledgements}

The authors would like to express their thanks to Brazilian Research Agencies CNPq, CAPES and FINEP for supporting this work, and are also grateful to the authors of the references that helped in the improvement of quality of this paper.

\section{Conflicts of Interest}

The authors declare no conflicts of interest regarding the publication of this paper.

\section{References}

[1] Sahu, B.K. and Subudhi, B. (2014) The State of Art of Autonomous Underwater Vehicles in Current and Futures Decades. Proceedings of First International Conference on Automation, Control, Energy and Systems, Hooghly, 1-2 February 2014, 1-6. https://doi.org/10.1109/ACES.2014.6808014

[2] Jain, S.K., Mohammad, S., Bora, S. and Singh, M. (2015) A Review Paper on: Autonomous Underwater Vehicle. International Journal of Scientific \& Engineering Re search, 6, 38-40.

[3] Dantas, J.L.D. and Barros, E.A. (2013) Numerical Analysis of Control Surface Effects on AUV Manoeuvrability. Applied Ocean Research, 42, 168-181. https://doi.org/10.1016/j.apor.2013.06.002

[4] Vasudev, K.L., Sharma, R. and Bhattacharyya, S.A. (2014) A CAGD+CFD Inte- 
grated Optimization Model for Design of AUVs. Proceedings of Oceans, Taipei, China, 7-10 April 2014, 1-8. https://doi.org/10.1109/OCEANS-TAIPEI.2014.6964557

[5] Sousa, J.V.N., Macêdo, A.R.L., Amorim Junior, W.F. and Lima, A.G.B. (2014) Numerical Analysis of Turbulent Fluid Flow and Drag Coefficient for Optimizing the AUV Hull Design. Open Journal of Fluid Dynamics, 4, 263-277. https://doi.org/10.4236/ojfd.2014.43020

[6] Mansoorzadeh, S. and Javanmard, E. (2014) An Investigation of Free Surface Effects on Drag and Lift Coefficients of an Autonomous Underwater Vehicle (AUV) Using Computational and Experimental Fluid Dynamics Methods. Journal of Fluids and Structures, 51, 161-171. https://doi.org/10.1016/j.jfluidstructs.2014.09.001

[7] Gao, T., Wang, Y., Pang, Y. and Cao, J. (2016) Hull Shape Optimization for Autonomous Underwater Vehicles Using CFD. Engineering Applications of Computational Fluid Mechanics, 10, 599-607. https://doi.org/10.1080/19942060.2016.1224735

[8] Tian, W., Mao, Z., Zhao, F. and Zhao, Z. (2017) Layout Optimization of Two Autonomous Underwater Vehicles for Drag Reduction with a Combined CFD and Neural Network Method. Complexity, 2017, Article ID: 5769794. https://doi.org/10.1155/2017/5769794

[9] Aymen, M., Hedi, K., Abid, M.S. and Zied, D. (2017) Numerical Study of Attack's Angle Effect on Drag Coefficient of AUV Hull Design. American Journal of $\mathrm{Me}$ chanical Engineering, 5, 8-13.

[10] Aymen, M., Hedi, K., Abid, M.S. and Zied, D. (2018) Impact of Stern Design on Hydrodynamic Drag of AUV's Hull. Indian Journal of Geo Marine Sciences, 47, 89-95.

[11] Myring, D.F. (1976) A Theoretical Study of Body Drag in Subcritical Axisymmetric Flow. Aeronautical Quarterly, 27, 186-194. https://doi.org/10.1017/S000192590000768X

[12] Jagadeesh, P., Murali, K. and Idichandy, V.G. (2009) Experimental Investigation of Hydrodynamic Force Coefficients over AUV Hull Form. Ocean Engineering, 36, 113-118. https://doi.org/10.1016/j.oceaneng.2008.11.008

[13] Rawson, K.J. and Tupper, E.C. (2011) Basic Ship Theory-Volume 2. 5th Edition, Butterworth-Heinemann, Oxford.

[14] Ansys Inc. (2014) CFX-Theory Manual (Release 15.0). Canonsburg.

[15] Kaviany, M. (2001) Principles of Convective Heat Transfer. 2nd Edition, Springer, New York. https://doi.org/10.1007/978-1-4757-3488-1

[16] Warsi, Z.U.A. (1999) Fluid Dynamics: Theoretical and Computational Approaches. 2nd Edition, CRC Press, Boca Raton, FL. 